ISSN 0103-5150

Fisioter. Mov., Curitiba, v. 25, n. 1, p. 231-239, jan./mar. 2012 Licenciado sob uma Licença Creative Commons

\title{
Efetividade do exercício físico na insuficiência renal crônica
}

\author{
Effectiveness of physical exercise in chronic renal failure
}

\author{
Leilane Cristielle de Alencar Nascimento $^{[\mathrm{a}]}$, Érika Bona Coutinhoo ${ }^{[\mathrm{b}]}$, Kelson Nonato Gomes da Silva ${ }^{[\mathrm{c}]}$ \\ [a] Aluna do curso de Fisioterapia da Universidade Estadual do Piauí (UESP), Teresina, PI - Brasil, e-mail: \\ l.cnascimento@hotmail.com \\ [b] Mestre em Bioengenharia pela Universidade do Vale do Paraíba (UNIVAP); Especialista em Terapia Manual e Postural pela \\ CESUMAR; Especialista em Terapia Manual e Postural pelo Centro Universitário de Maringá, Maringá, PR - Brasil. \\ [c] Doutorando em Ciências - Medicina Interna e Terapêutica pela Universidade Federal de São Paulo (UNIFESP/EPM), \\ Teresina, PI - Brasil, e-mail: kelson_fisio@hotmail.com
}

\section{Resumo}

Introdução: 0 exercício físico em nefropatas submetidos à hemodiálise se faz importante, uma vez que esses pacientes apresentam acentuada redução da funcionalidade e do condicionamento, o que interfere negativamente na qualidade de vida. Contudo, os benefícios, o tipo de exercício mais adequado e parâmetros como intensidade, frequência e duração não estão bem esclarecidos para essa população. Objetivos: Realizar uma revisão de literatura sobre a influência do exercício físico em pacientes renais crônicos submetidos à hemodiálise. Materiais e métodos: Foram realizadas buscas nas bases MEDLINE, LILACS, PEDro, SciELO e PubMed, sendo selecionados artigos (ensaios clínicos controlados randomizados, séries de casos e estudos de caso) nos idiomas inglês e português, publicados entre 2000 e 2010. Resultados: Foram encontrados 105 artigos, sendo 82 da base de dados PubMed, 16 da base PEDro e 7 da base SciELO. Não foram encontrados artigos nas bases MEDLINE e LILACS. Desses, apenas sete preenchiam aos critérios de inclusão. A partir desses sete artigos, realizou-se busca manual ativa na lista de referências dessas publicações, nas quais foi possível verificar a presença de apenas três referências. Conclusão: Pôde-se concluir, por meio dos artigos revisados, que os exercícios físicos, seja aeróbico e/ou de resistência, possuem efeitos incrementais na capacidade funcional, função 
muscular e qualidade de vida de nefropatas submetidos à hemodiálise. Portanto, o treinamento físico deve ser considerado como uma modalidade terapêutica importante, sendo fundamental a inserção do fisioterapeuta nos centros dialíticos, fazendo parte de uma equipe multidisciplinar.

Palavras-chave: Hemodiálise. Exercício aeróbico. Qualidade de vida.

\begin{abstract}
Introduction: Physical activity in nephropathy patients submitted to hemodialysis becomes important since these patients presents marked reduction in the functionality and conditioning that impairs life quality. However, the benefits, the most appropriate type of exercise and parameters such as intensity, frequency and duration are not well defined in this population. Objectives: To review the literature about the effects of physical exercise in chronic renal patients submitted to hemodialysis. Materials and methods: MEDLINE, LILACS, PEDro, PubMed and SciELO bases were searched, being selected articles (clinical randomized controlled trials, series of case and case study) in languages, English and Portuguese, published between 2000 and 2010. Results: 105 articles were found, 82 from PubMed database, 16 from PEDro and 7 from SciELO base. No articles were found in MEDLINE and LILACS bases. From these ones, only seven fulfilled the inclusion criteria. From these seven articles, an active manual search was held in the list of references of these publications, being possible to verify in them the presence of only three references. Conclusion: By the reviewed articles it was possible to conclude that either aerobic and/or physical exercise have incremental effects on functional capacity, muscle function and life quality of nephropathy patients submitted to hemodialysis. Therefore, physical training should be considered as an important therapeutic modality, being central the insertion of the physiotherapist in dialysis centers as part of a multidisciplinary team.
\end{abstract}

Keywords: Hemodialysis. Aerobic exercise. Quality of life.

\section{Introdução}

A doença renal crônica (DRC) é uma lesão dos rins, provocada por uma variedade de nefropatias, que evolui com perda lenta, progressiva e irreversível, das suas múltiplas funções (1-3). Ela está associada à elevada morbimortalidade, sendo que a hipertensão arterial e o diabetes mellitus são suas principais causas (2-6). Em sua fase mais avançada, quando os rins não conseguem manter a normalidade do meio interno, é definida como insuficiência renal crônica (IRC). Nessa fase, o paciente encontra-se intensamente sintomático, desenvolvendo completamente a síndrome urêmica, cujas principais manifestações são: irritabilidade, tremores, polineuropatia e miopatia urêmica, náuseas, hipertensão arterial, insuficiência cardíaca e anemia $(7,2)$.

No Brasil, o número de pacientes em terapia renal substitutiva cresce a taxas de $8 \%$ ao ano (8). Assim, a DRC se configura em uma questão de saúde pública, na medida em que aumentam a incidência e a prevalência de pessoas em programas dialíticos. Segundo dados do Ministério da Saúde em 2009, cerca de 90.000 pessoas foram submetidas a tratamento dialítico, gerando despesa anual em torno de um bilhão de reais em recursos do Sistema Único de Saúde (9).

0 tratamento de escolha substitutivo da função renal mais utilizado é a hemodiálise (HD), sendo indicado principalmente com base na filtração glomerular e quadro clínico do paciente $(2,7,10-12)$. Essa intervenção é geralmente realizada três vezes por semana, três a quatro horas por sessão $(11,10)$ e, apesar de prolongar substancialmente a sobrevida dos pacientes, estudos têm demonstrado que indivíduos com IRC submetidos à HD apresentam fraqueza muscular, anemia, cardiopatia, depressão, hipertensão arterial, alterações metabólicas e respiratórias, entre outros distúrbios, levando à redução progressiva na funcionalidade e no condicionamento, além de interferir de maneira negativa na qualidade de vida (QV) desses pacientes $(10,1,13)$.

Por conta dessas manifestações, o tratamento da IRC também deve incluir reabilitação física. Cada vez mais, estudos revelam que a fisioterapia, inter ou intradialítica, é parte integrante dessa reabilitação, 
pois contribui de forma significativa na prevenção, no retardo da evolução e na melhoria de várias complicações apresentadas pelo paciente renal (14-16).

No entanto, a aplicação de programas de exercícios no doente renal crônico, na prática clínica, ainda não se tornou rotina (17). Segundo a Diretriz de Reabilitação Cardiopulmonar e Metabólica (2006), tem sido demonstrada a importância do exercício físico para essa população, inclusive os submetidos a programas de HD, os quais apresentam acentuada redução da capacidade cardiorrespiratória (18). Contudo, os benefícios do treinamento físico, o tipo de exercício mais adequado e parâmetros como intensidade, frequência e duração não estão bem esclarecidos nessa população (3).

0 presente estudo tem como objetivo avaliar a influência do exercício físico em pacientes renais crônicos submetidos à HD.

\section{Metodologia}

Foram realizadas buscas nas bases de dados MEDLINE, LILACS, PEDro, SciELO e PubMed, sendo selecionados artigos (ensaios clínicos controlados randomizados, séries de casos e estudos de caso) nos idiomas inglês e português, publicados no período entre 2000 e 2010, que abordaram o tratamento de hemodiálise com duração superior a três meses em indivíduos adultos (maiores de 18 anos de idade), em uso ou não de eritropoetina, e que avaliaram os desfechos dos programas de exercícios em relação à qualidade de vida, força muscular, capacidade funcional e pressão arterial. Além disso, analisamos as referências bibliográficas dos artigos selecionados e incluímos os artigos relevantes. A estratégia de busca foi realizada e adaptada para as bases de dados com base nos seguintes descritores: hemodialysis OR "renal insufficiency chronic" AND "exercise aerobic" OR "muscle strength" OR "quality of life".

\section{Resultados}

Após realizar a busca nas bases de dados mencionadas anteriormente, foram encontrados 105 artigos, sendo 82 da base de dados PubMed, 16 da base PEDro e 7 da base SciELO. Não foram encontrados artigos nas bases MEDLINE e LILACS. Desses, apenas sete preenchiam aos critérios de inclusão. A partir desses sete artigos realizou-se busca manual ativa na lista de referências dessas publicações, nas quais foi possível verificar a presença de apenas três referências sobre o conteúdo anteriormente abordado. Podemos encontrar o resumo dos 10 artigos nos Quadros 1 e 2.

\section{Discussão}

Os estudos selecionados serão discutidos de acordo com as variáveis analisadas.

\section{Pressão arterial}

As complicações cardiovasculares constituem a principal causa de morte em pacientes portadores de IRC submetidos à HD $(27,6)$. Mais de $80 \%$ dos pacientes com doença renal em estágio avançado são acometidos pela hipertensão arterial, sendo que $40 \%$ a $50 \%$ destes permanecem hipertensos mesmo após o início da diálise (27).

Vários fatores contribuem na fisiopatologia desse distúrbio em renais crônicos, com destaque para: a retenção de sódio e água, a hiperatividade do sistema nervoso simpático e do sistema renina angiotensina aldosterona, o uso de eritropoietina recombinante e o hiperparatireoidismo secundário $(5,27)$.

Apesar do treino aeróbico ser aplicado como tratamento coadjuvante na redução dos níveis pressóricos em hipertensos (28) os efeitos do exercício físico no controle pressórico em pacientes em HD não estão bem esclarecidos (22).

Reboredo et al. (24) submeteram um grupo de 14 pacientes à monitorização ambulatorial da pressão arterial com o objetivo de avaliar o efeito do treinamento aeróbico, realizado durante a HD, no controle da pressão arterial. Os resultados mostraram redução significativa da pressão arterial sistólica de $151 \pm 18,4 \mathrm{mmHg}$ para $143 \pm 14,7 \mathrm{mmHg}$, da pressão arterial diastólica de $94 \pm 10,5 \mathrm{mmHg}$ para $91 \pm 9,6 \mathrm{mmHg}$ e da pressão arterial média de $114 \pm$ $13,0 \mathrm{mmHg}$ para $109 \pm 11,4 \mathrm{mmHg}$, antes e após 12 semanas de treino, respectivamente.

Por outro lado Molsted et al. (22) em um estudo controlado randomizado avaliaram, por um período de cinco meses, o efeito de $1 \mathrm{~h}$ de exercícios aeróbicos e de força, realizados duas vezes por semana, 
Quadro 1 - Resumo apenas dos ensaios clínicos controlados randomizados (ECCR)

\begin{tabular}{|c|c|c|c|c|c|c|c|}
\hline \multirow{2}{*}{$\begin{array}{l}\text { Autor/ano/ } \\
\text { tipo de estudo }\end{array}$} & \multirow{2}{*}{$\begin{array}{l}\text { Paciente/ } \\
\text { grupos }\end{array}$} & \multirow[t]{2}{*}{ Modalidade } & \multirow{2}{*}{$\begin{array}{l}\text { Frequência/ } \\
\text { duração/ intensidade }\end{array}$} & \multicolumn{4}{|c|}{ Melhora nos desfechos avaliados } \\
\hline & & & & PA & FM & CF & QV \\
\hline $\begin{array}{l}\text { Ouzouni S, et al., } \\
2009 \text { (19) / ECCR }\end{array}$ & $\begin{array}{l}\text { Gl:20 (exerc DHD) } \\
\text { GC: } 15 \text { (só HD) }\end{array}$ & $\begin{array}{l}\text { Gl: } 30 \text { 'de CEM }+30 \text { 'de } \\
\text { exerc de fortalecimento } \\
\text { com pesos e Therabands. }\end{array}$ & $\begin{array}{l}3 \times / \text { sem/ } 10 \text { meses } \\
13-14 \text { Escala de Borg }\end{array}$ & NA & NA & $S$ & S \\
\hline $\begin{array}{l}\text { Cheema B, et al., } \\
2007 \text { (20) / ECCR }\end{array}$ & $\begin{array}{l}\text { Gl: } 24 \text { (Exerc DHD) } \\
\text { GC:25 }\end{array}$ & $\begin{array}{l}\text { Gl: exerc com pesos e } \\
\text { therabands de MMSS e } \\
\text { MMII }\end{array}$ & $\begin{array}{l}3 \mathrm{x} / \mathrm{sem} / 12 \mathrm{sem} \\
15 \text { a } 17 \text { na Escala de } \\
\text { Borg }\end{array}$ & NA & $S$ & NA & $S$ \\
\hline $\begin{array}{l}\text { Storer T, et al., } \\
2005 \text { (14) / ECCR }\end{array}$ & $\begin{array}{l}\text { Gl: } 12 \text { (exerc DHD) } \\
\text { GC: } 12 \text { (só HD) } \\
\text { GS:12 }\end{array}$ & Gl: 40’ de CEM de MMII & $\begin{array}{l}3 \mathrm{x} / \mathrm{sem} / 10 \mathrm{sem} \\
50 \% \mathrm{VO}_{2} \text { máx }\end{array}$ & NA & $S$ & $S$ & NA \\
\hline $\begin{array}{l}\text { Vilsteren M, et al., } \\
2005 \text { (21) } \\
\text { ECCR }\end{array}$ & $\begin{array}{l}\text { Gl: } 53 \text { (exerc de } \\
\text { força no período de } \\
\text { pré-diálise e treino } \\
\text { aeróbio em CEM } \\
\text { durante a diálise). } \\
\text { GC:43(só HD) }\end{array}$ & $\begin{array}{l}\text { Gl: } 20 \text { ' exerc de resistên- } \\
\text { cia com baixo peso + } \\
20-30^{\prime} \text { em CEM }\end{array}$ & $\begin{array}{l}\text { Mínimo 2x/sem/12 sem } \\
12 \text { na escala de Borg }\end{array}$ & NA & $S$ & N & $S$ \\
\hline $\begin{array}{l}\text { Molsted S, et al., } \\
2004 \text { (22) } \\
\text { ECCR }\end{array}$ & $\begin{array}{l}\text { Gl: 22(exerc DHD) } \\
\text { GC:11 (só HD) }\end{array}$ & $\begin{array}{l}\text { Gl: } 20-30 \text { 'de exerc de } \\
\text { força }+15-20 \text { 'de CEM. }\end{array}$ & $\begin{array}{l}2 x / \text { sem/ } 5 \text { meses } \\
14-17 \text { na Escala de } \\
\text { Borg }\end{array}$ & N & NA & $S$ & S \\
\hline $\begin{array}{l}\text { Depaul V, et al., } \\
2002(23) \\
\text { ECCR }\end{array}$ & $\begin{array}{l}\text { Gl: } 20 \text { (exerc DHD) } \\
\text { GC: } 18 \text { (exerc DHD) } \\
\text { GS: (?) }\end{array}$ & $\begin{array}{l}\text { Gl: } 20 \text { ' de treino aeróbico } \\
\text { em CEM + exerc resisti- } \\
\text { dos do quadríceps e IQT, } \\
\text { GC: } 30^{\prime} \text { de exerc de ADM } \\
\text { de baixa intensidade }\end{array}$ & $\begin{array}{l}\text { 3x/sem /12 sem } \\
\text { Gl: 80\% da FCmáx.; } \\
\text { 50\% de 5-RM sendo } \\
\text { a carga aumentada } \\
\text { gradualmente }\end{array}$ & NA & $S$ & $\begin{array}{l}\mathrm{N}= \\
\mathrm{TC6} \\
\mathrm{S}= \\
\text { TE }\end{array}$ & N \\
\hline
\end{tabular}

Legenda: $\mathrm{ECCR}=$ ensaio clínico controlado randomizado; $\mathrm{GC}=$ grupo controle; $\mathrm{Gl}$ = grupo intervenção; $\mathrm{GS}$ = grupo de indivíduos saudáveis; $\mathrm{CEM}=$ cicloergômetro; $\mathrm{DHD}$ = durante a diálise; sem = semana; $\mathrm{IQT}=$ isquiotibiais; Exerc = exercícios; $H \mathrm{HD}=$ Hemodiálise; ADM = amplitude de movimento; RM = repetição máxima; MMII = membros inferiores; MMSS = membros superiores; TC6 $^{\prime}$ = teste de caminhada de 6 minutos; V02 máx = consumo máximo de oxigênio; TE = teste ergométrico; $\mathrm{PA}=$ pressão arterial; $\mathrm{FM}=$ função muscular; $\mathrm{CF}=$ capacidade funcional; $\mathrm{QV}=$ qualidade de vida; $\mathrm{S}=\mathrm{SIM} ; \mathrm{N}=\mathrm{NÃ0}$; NA = não avaliado.

Fonte: Dados da pesquisa.

com intensidade de 4-17 na escala de Borg e concluíram não haver melhora da pressão arterial. Esse resultado pode ser explicado, de acordo com o autor, pelo curto período de intervenção,poucas sessões de exercício por semana, ou um método impreciso para aferir a pressão arterial.

\section{Função muscular}

A perda de massa muscular é o mais significante preditor de mortalidade nos pacientes em HD (29). A musculatura se atrofia e como consequência, ocorre no organismo uma fraqueza generalizada, causada pela perda de força, que comparada a de indivíduos normais é de 30 a 40\% menor, levando o paciente ao descondicionamento físico $(1,13)$. 0 treino físico é um importante fator no controle e reversão dessa perda, apesar de ainda não estarem totalmente compreendidos os efeitos do mesmo nesta população (30).

Neste sentido, Cantareli et al. (15) aplicaram 5 meses de treinamento de força e resistência durante a HD , demonstrando aumento da força muscular para os extensores do joelho sendo a média das cargas tolerada por MID: $4,71 \pm 3,03$ vs $6,07 \pm$ 
Quadro 2 - Resumo dos artigos selecionados não randomizados

\begin{tabular}{|c|c|c|c|c|c|c|c|}
\hline \multirow{2}{*}{$\begin{array}{l}\text { Autor/Ano/ } \\
\text { Tipo de estudo }\end{array}$} & \multirow{2}{*}{$\begin{array}{l}\text { Paciente/ } \\
\text { grupos }\end{array}$} & \multirow[t]{2}{*}{ Modalidade } & \multirow{2}{*}{$\begin{array}{l}\text { Frequência/ } \\
\text { duração/ intensidade }\end{array}$} & \multicolumn{4}{|c|}{ Melhora nos desfechos avaliados } \\
\hline & & & & PA & FM & $\mathrm{CF}$ & QV \\
\hline $\begin{array}{l}\text { Reboredo M, et al., } \\
2010 \text { (24) } \\
\text { EE }\end{array}$ & $\begin{array}{l}14 \text { pacientes (exerc } \\
\text { DHD) }\end{array}$ & $\begin{array}{l}\text { Alongamentos passivos } \\
\text { de MMII e } 30^{\prime} \text { de CEM . }\end{array}$ & $\begin{array}{l}3 \mathrm{x} / \mathrm{sem} / 12 \text { sem } \\
11-13 \text { na Escala de } \\
\text { Borg }\end{array}$ & S & NA & $\begin{array}{l}\mathrm{N}-\mathrm{VO}_{2} \\
\text { máx } \\
\mathrm{S}-\mathrm{TC6}\end{array}$ & NA \\
\hline $\begin{array}{l}\text { Cantareli F, et al., } \\
2009 \text { (15) } \\
\text { EC }\end{array}$ & $\begin{array}{l}7 \text { pacientes (exerc } \\
45 \text { 'após iniciar } \\
\text { a diálise e } 45 \text { ' } \\
\text { antes de finalizar a } \\
\text { sessão de HD }\end{array}$ & $\begin{array}{l}\text { Exerc isométricos, } \\
\text { isotônicos e isotônicos } \\
\text { livres de carga de MMII, } \\
\text { além dos abdominais e } \\
\text { glúteos }\end{array}$ & $\begin{array}{l}2 x / \text { sem } / 5 \text { meses } \\
50 \% \text { de } 1 \mathrm{RM}\end{array}$ & NA & S & N & $\mathrm{N}$ \\
\hline $\begin{array}{l}\text { Sakkas K, et al., } \\
2003(25) \\
\text { EENC }\end{array}$ & $\begin{array}{l}\text { Grupo A: } \\
12 \text { (DP + exerc) } \\
\text { Grupo B: } \\
12 \text { (exerc DHD) }\end{array}$ & GB: 40’ CEM de MMII & $\begin{array}{l}3 \mathrm{x} / \mathrm{sem} / \mathrm{6} \text { meses } \\
90 \% \text { do } \mathrm{VO}_{2} \text { máx }\end{array}$ & NA & $S$ & NA & NA \\
\hline $\begin{array}{l}\text { Painter P, et al., } \\
2000(26) \\
\text { EEC }\end{array}$ & $\begin{array}{l}\text { Total: } 286 \\
\text { Gl: Exerc DHD } \\
\text { GC: só HD }\end{array}$ & $\begin{array}{l}\text { Gl: } 8 \text { sem de exerc } \\
\text { domiciliar }+8 \text { sem de } \\
\text { 30' de CEM de MMII }\end{array}$ & $\begin{array}{l}\text { Pelo menos 3x/sem / } 16 \\
\text { sem individualizada com } \\
\text { aumento progressivo da } \\
\text { carga e duração }\end{array}$ & NA & NA & $S$ & S \\
\hline
\end{tabular}

Legenda: $\mathrm{EE}=$ estudo experimental; $\mathrm{EC}=$ ensaio clínico; $\mathrm{EENC}=$ estudo experimental não controlado; $\mathrm{EEC}$ = estudo experimental controlado; $\mathrm{GC}=$ grupo controle; $\mathrm{Gl}$ = grupo intervenção; $\mathrm{CEM}=$ cicloergômetro; $\mathrm{DHD}=$ durante a diálise; $\mathrm{DP}=$ diálise peritoneal; sem = semana; Exerc = exercícios; $\mathrm{HD}$ = hemodiálise; MMII = membros inferiores; TC6' = teste de caminhada de 6 minutos; $\mathrm{VO}_{2}$ máx = consumo máximo de oxigênio; $\mathrm{PA}$ = pressão arterial; $\mathrm{FM}$ = função muscular; $\mathrm{CF}=$ capacidade funcional; $\mathrm{QV}=$ qualidade de vida; $S$ = SIM; $N$ = NÃ0; NA = não avaliado.

Fonte: Dados da pesquisa.

2,62 vs $8,42 \pm 3,30 \mathrm{~kg}$; MIE $4,85 \pm 3,13$ vs $6,21 \pm 2,82$ vs $8,57 \pm 3,99 \mathrm{~kg}, \mathrm{p}<0,05$. Outros autores $(14,20$, $21,23)$, estudaram o efeito do treinamento aeróbio e/ou de resistência encontrando também melhora significativa na força muscular.

A atrofia de fibras musculares tipo I e II, particularmente as do tipo IIB, são fatores importantes que, juntamente com as alterações histoquímicas como baixa concentração de enzimas aeróbicas, baixa capacidade oxidativa, perda da capilaridade e baixos níveis de proteínas contráteis contribuem para o quadro de disfunção muscular $(31,32)$.

Para verificar os efeitos do exercício nas alterações musculares presentes em pacientes renais crônicos em HD, Sakkas et al. (25) analisaram a morfologia do músculo gastrocnêmio de doze pacientes antes e após um programa de exercício aeróbico realizado três vezes por semana durante 6 meses. Os resultados evidenciaram que o treinamento proposto melhorou o trofismo muscular, aumentou a área de secção transversa em $46 \%$ e reduziu a atrofia das fibras musculares tipo I (51\% para $15 \%$ ), tipo IIA (58\% para $21 \%$ ) e tipo IIB (62\% para $32 \%$ ). Além disso, diferenças significativas foram encontradas com relação ao aumento da capilarização muscular.

\section{Capacidade funcional}

Muitos estudos demonstram que pacientes portadores de DRC sob tratamento hemodialítico apresentam redução da capacidade funcional, sendo que a capacidade de exercício pode ser $50 \%$ menor em relação a de indivíduos saudáveis (33, 
34). Vários fatores estão associados a essa redução, entre eles a diminuição da atividade física, fraqueza muscular, anemia, disfunção ventricular, controles metabólico e hormonal anormais (30). Atualmente, grande interesse vem sendo atribuído à avaliação da capacidade funcional desses pacientes por meio do teste de caminhada de seis minutos (TC6') e outros testes como o teste de sentar-e-levantar (35). Estes testes são simples, mensuram a capacidade funcional por meio de informações básicas, e fornecem dados importantes para acompanhar a evolução do paciente no decorrer da doença avaliando assim os benefícios de programas de reabilitação $(35,36)$.

Em estudo realizado por Reboredo et al. (24), 14 pacientes renais crônicos em HD foram submetidos ao treino aeróbico, ao final de 12 semanas a distância percorrida no TC6' aumentou em 10\%. Além disso, os pacientes apresentaram incremento de 35\% no tempo de tolerância ao exercício aeróbico, achados indicativos do benefício de um programa de exercício supervisionado na melhora da capacidade funcional. Corroborando esses resultados Painter et al. (26), em estudo controlado de 16 semanas de exercício aeróbico individualizado, demonstraram que houve aumento no tempo da velocidade da marcha normal, melhora do teste sentar-levantar e TC6'.

Já, DePaul et al. (23), em estudo controlado randomizado envolvendo treinamento aeróbico associado ao fortalecimento muscular não encontraram valores significativos para o TC6' sendo que, comparando com a amostra de saudáveis, os pacientes estavam em funcionamento significativamente inferior ( $464 \pm 94$ vs. $508 \pm 59$ respectivamente). Essas diferenças no desempenho durante o TC6' podem ser atribuídas à grande diversidade nas características fisiopatológicas, clínicas, terapêuticas e físico-funcionais identificadas nessa população (35).

0 consumo máximo de $02\left(\mathrm{VO}_{2}\right.$ máx) é considerado o melhor índice de capacidade funcional (3638). Pacientes com IRC podem apresentar $\mathrm{VO}_{2}$ máx com valores entre 15,3 e $21 \mathrm{~mL} / \mathrm{kg} / \mathrm{min}$, o que é somente metade do observado em indivíduos normais sedentários (1). 0 treinamento físico pode melhorar este parâmetro aumentando a sobrevida desses pacientes (38). Em estudo realizado por Storer et al. (14), 12 pacientes renais crônicos em HD foram submetidos ao exercício aeróbico, 3 vezes por semana durante 10 semanas, com objetivo de avaliar o efeito desta intervenção na capacidade funcional por meio do $\mathrm{VO}_{2}$ máx. Observou-se au-

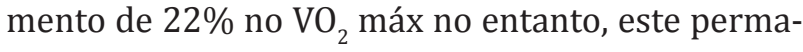
neceu $30 \%$ abaixo daquele do grupo de indivíduos saudáveis. Os autores afirmam que é possível que o exercício físico em curto prazo, com baixa intensidade, em indivíduos com IRC avançada e outras comorbidades, não seja suficiente para restabelecer função aeróbica ao nível observado em indivíduos saudáveis. Ouzouni et al. (19) também observaram um aumento (21\%) do $\mathrm{VO}_{2}$ máx.

Esses achados contrapõe-se aos resultados de Vilsteren et al. (21) e Reboredo et al. (24) que não encontraram efeitos significativos sobre o V02 máx após treino aeróbico. De acordo com os autores essas discrepâncias podem estar relacionadas a dificuldades técnicas à realização do exame, assim como idade e comorbidades dos pacientes avaliados.

\section{Qualidade de vida}

Pesquisas demonstram que a IRC e o tratamento hemodialítico estão entre as patologias e terapias que mais afetam a QV pois levam a uma limitação da capacidade cardiorrespiratória e física, o que pode prejudicar o desempenho nas atividades de lazer, trabalho e convívio social $(33,39,40)$. Estudos demostram que a atividade física pode contribuir para uma melhora da QV de pacientes com IRC $(15,40)$.

Painter et al. (26) verificaram o efeito de um programa constituído de 8 semanas de exercícios domiciliares seguidos de 8 semanas de cicloergômetro durante a diálise na $\mathrm{QV}$ de pacientes renais crônicos. Após quatro meses, os autores observaram ganho significativo nas seguintes dimensões do SF-36: capacidade funcional $(47,7 \pm$ $28,3$ vs. $53,4 \pm 27 \mathrm{p}=0,004)$, aspectos físicos $(40,4$ $\pm 40,3$ vs. $54,5 \pm 21,4 \mathrm{p}<0,001)$, dor $(60,5 \pm 28,1$ vs. $66,6 \pm 28,6 \mathrm{p}=0,003$ ) e estado geral de saúde ( $45 \pm 21,9$ vs. $49,1 \pm 22,5 \mathrm{p}=0,05$ ). De acordo com os autores os resultados do SF-36 claramente indicam que a atividade física específica afetam os aspectos físicos da saúde, porque não houve mudanças nos escores de saúde mental em qualquer grupo de pacientes. Em concordância, Vilsteren et al. (21) em 12 semanas de treinamento aeróbico intradialíse observaram mudança significativa nos componentes Vitalidade e Estado geral da saúde do SF-36 ( $p=0,001)$. 
No entanto Cantareli et al. (15) em seu ensaio clínico com sete pacientes demonstraram que as médias dos valores das dimensões aspectos físicos, dor, estado geral de saúde e vitalidade aumentaram após o treinamento muscular, porém não apresentaram significância.

\section{Considerações finais}

Pôde-se concluir, por meio dos artigos revisados, que o exercício físico, seja aeróbico e/ou de resistência, possui efeitos incrementais na capacidade funcional, função muscular e qualidade de vida de pacientes com IRC submetidos a HD. No entanto não podemos concluir, por meio desta revisão, a real efetividade do treinamento físico sobre a variável cardiovascular pressão arterial devido à pequena quantidade de artigos encontrados e, além disso, nem todos apresentam uma boa qualidade metodológica.

Observou-se também, nos estudos analisados, uma grande diversidade quanto aos protocolos de treinamento utilizados em termos de intensidade, frequência e duração, o que demonstra que esses parâmetros devem se adequar às características físicas e condições clínicas de cada paciente. Além disso, os estudos não possuem a mesma metodologia, sendo necessário o desenvolvimento de estudos de maior qualidade metodológica utilizando grupos de pacientes mais homogêneos, principalmente com relação à idade e características clínicas.

Portanto, o treinamento físico deve ser considerado como uma modalidade terapêutica importante, sendo fundamental a inserção do fisioterapeuta nos centros dialíticos, fazendo parte de uma equipe multidisciplinar.

\section{Referências}

1. Soares A, Zehetmeyer M, Rabuske M. Atuação da fisioterapia durante a hemodiálise visando à qualidade de vida do paciente renal crônico. Rev de Saúde da UCPEL. 2007;1(1):7-12.

2. Draibe SA, Cendoroglo M. Tratamento conservador da insuficiência renal crônica. Diagnóstico \& Tratamento. 2001;6(2):17-23.
3. Najas CS, Pissulin FDM, Pacagnelli FL, Betonico GN, Almeida IC, Neder JA. Segurança e eficácia no treinamento físico na insuficiência renal crônica. Rev Bras Med Esporte. 2009;15(5):384-88.

4. Marques BA, Pereira CD, Ribeiro R. Motivos e freqüência de internação dos pacientes com IRC em tratamento hemodialítico. Arq Ciênc Saúde. 2005; 12(2):67-72.

5. Martin LC, Barretti P, Kochi AC, Balbi AL, Caramori JS, Franco RJ, et al. Fisiopatologia da Hipertensão Arterial em Diálise. J Bras Nefrol. 2006;28(4):225-31.

6. Salgado Filho N, Brito DJA. Doença renal crônica: a grande epidemia deste milênio. J Bras Nefrol. 2006; 28(3 supl. 2):1-5.

7. Romão JE, Jr. Doença renal crônica: definição, epidemiologia e classificação. J Bras Nefrol. 2004;26(suppl 1):1-3.

8. Sesso R, Lopes, AA, Thomé AS, Belilacqua, JL, Romão JE, Jr, Lugon J. Relatório do Censo Brasileiro de Diálise, 2008. J Bras Nefrol. 2008;30(4):233-8.

9. Brasil. Ministério da Saúde. Fundo Nacional de Saúde. Relatório anual 2005 [internet]. [acesso em 17 out 2010]. Disponível em: www.fns.saude.gov.br.

10. Rocha CBJ, Araújo S. Avaliação das pressões respiratórias máximas em pacientes renais crônicos nos momentos pré e pós-hemodiálise. J Bras Nefrol. 2010;32(1):107-13.

11. Terra FS, Costa AMDD, Figueiredo ET, Morais AM, Costa MD, Costa RD. As principais complicações apresentadas pelos pacientes renais crônicos durante as sessões de hemodiálise. Rev Bras Clin Med. 2010;8(3):187-92.

12. Barretti P. Indicações, escolha do método e preparo do paciente para a Terapia renal substitutiva (TRS), na Doença Renal Crônica (DRC). J Bras Nefrol. 2004;26(3 supl. 1):47-9.

13. Medeiros RH, Pinent CEC, Meyer F. Aptidão física de indivíduo com doença renal crônica. J Bras Nefrol. 2002;24(2):81-7.

14. Storer TW, Casaburi R, Sawelson S, Kopple JD. Endurance exercise training during haemodialysis improves strength, power, fatigability and physical performance in maintenance haemodialysis patients. Nephrol Dial Transplant. 2005;20(1):429-37. 
15. Cantareli F, Corrêa LB, Oliveira RN, Cunha LS. Efeito do treinamento muscular periférico na capacidade funcional e qualidade de vida nos pacientes em hemodiálise. J Bras Nefrol. 2009;31(1):18-24.

16. Seixas RJ, Giacomazzi CM, Figueiredo AEPL. Fisioterapia intradialítica na reabilitação do doente renal crônico. J Bras Nefrol. 2009;31(3):235-6.

17. Martins MRI, Cesarino CB. Atualização sobre programas de educação e reabilitação para pacientes renais crônicos submetidos à hemodiálise. J Bras Nefrol. 2004;26(1):45-50.

18. Diretriz de reabilitação cardiopulmonar e metabólica: aspectos práticos e responsabilidades. Arq Bras Cardiol. 2006;86(1):74-82.

19. Ouzouni S, Kouidi E, Sioulis A, Grekas D, Deligiannis A. Effects of intradialytic exercise training on health-related quality of life indices in haemodialysis patients. Clin Rehabil. 2009;23(1):53-63.

20. Cheema B, Abas H, Smith B, O'Sullivan A, Chan M, Patwardhan A, et al. Progressive exercise training for anabolism in kidney disease (PEAK): a randomized, controlled trial of resistance training during hemodialysis. J Am Soc Nephrol. 2007;18(15):94-601.

21. Vilsteren MCBA, Greef MHG, Huisman RM. The effects of a low-to-moderate intensity pre-conditioning exercise programme linked with exercise counseling for sedentary haemodialysis patients in The Netherlands: results of a randomized clinical trial. Nephrol Dial Transplant. 2005;20(1):141-6.

22. Molsted S, Eidemakb I, Sorensena HT, Kristensen JH. Five months of physical exercise in hemodialysis patients: effects on aerobic capacity, physical function and self-rated health. Nephron Clin Pract. 2004; 96(3):c76-c81.

23. DePaul V, Moreland J, Eager T, Clase CM. The effectiveness of aerobic and muscle strength training in patients receiving hemodialysis and EPO: a randomized controlled trial. Am J Kidney Dis. 2002;40(6):1219-29.

24. Reboredo MM, Henrique DMN, Chaoubah A, Paula RB. Treinamento aeróbico melhora a capacidade funcional de pacientes em hemodiálise crônica. Arq Bras Cardiol. 2010;94(6):823-8.
25. Sakkas GK, Sargeant AJ, Mercer TH, Ball D, Koufaki P, Karatzaferi C, et al. Changes in muscle morphology in dialysis patients after 6 months of aerobic exercise training. Nephrol Dial Transplant. 2003;18(9):1854-61.

26. Painter P, Carlson L, Carey S, Paul SM, Myll J. Physical functioning and healthrelated quality-of-life changes with exercise training in hemodialysis patients. Am J Kidney Dis. 2000;35(3):482-92.

27. Carmo WB, Almeida SC, Rezende FCM, Oliveira VK, Henriques DMN, Andrade LC, et al. Hipertensão arterial e hipertrofia ventricular esquerda em pacientes renais crônicos em tratamento hemodialítico. J Bras Nefrol. 2003;25:1-9.

28. Monteiro MF, Sobral DC, Filho. Exercício físico e o controle da pressão arterial. Rev Bras Med Esporte. 2004;10(6):513-6.

29. Cheema BS, Smith BC, Singh MA. A rationale for intradialytic exercise training as Standard clinical practice in ESRD. Am J Kidney Dis. 2005;45(5):912-6.

30. Coelho DM, Ribeiro JM, Soares DD. Exercícios físicos durante a hemodiálise: uma revisão sistemática. J Bras Nefrol. 2008;30(2):88-98.

31. Moreira PR, Barros E. Atualização em fisiologia e fisiopatologia renal: bases fisiopatológicas da miopatia na insuficiência renal crônica. J Bras Nefrol. 2000;22(1):40-4.

32. Coelho MC, Godoy CG, Tavares H, Navarro F, Almeida AL. Avaliação funcional e prescrição de treinamento para paciente portador de insuficiência renal crônica submetido a hemodiálise: um relato de caso. Revista Brasileira de Prescrição e Fisiologia do Exercício. 2007;1(3):29-41.

33. Cunha MS, Andrade V, Guedes CAV, Meneghetti CHZ, Aguiar AP, Cardoso AL. Avaliação da capacidade funcional e da qualidade de vida em pacientes renais crônicos submetidos a tratamento hemodialítico. Fisioter Pesq. 2009;16(2):155-60.

34. Jatobá JPC, Amaro WF, Andrade APA, Cardoso FPF, Monteiro AMH, Oliveira MAM. Avaliação da função pulmonar, força muscular respiratória e teste de caminhada de seis minutos em pacientes portadores de doença renal crônica em hemodiálise. J Bras Nefrol. 2008;30(4):280-7. 
35. Dipp T, Antônio SMV, Signori LU, Strimban TM, Nicolodi G, Sbruzzi G, et al. Força muscular respiratória e capacidade funcional na Insuficiência renal terminal. Rev Bras Med Esporte. 2010 [acesso 20 jun. 2010];16(4). Disponível em: http://www.scielo.br/scielo.php?pid $=$ S1517-86922010000400002\&script=sci_arttext.

36. Cury JL, Brunetto AF, Aydos RD. Efeitos negativos da insuficiência renal crônica sobre a função pulmonar e a capacidade funcional. Rev Bras Fisioter. 2010;14(2):91-8.

37. Moreira PR. Resposta cronotrópica ao exercício em pacientes com insuficiência renal crônica em programa de hemodiálise [tese]. Porto Alegre: Universidade Federal do Rio Grande do Sul; 2003.

38. Adams GR, Nosratola DV. Skeletal muscle dysfunction in chronic renal failure: effects of exercise. Am J Physiol Renal Physiol. 2006;290(4):F753-61.
39. Barbosa LMM, Andrade MP, Jr, Bastos KA. Preditores de qualidade de vida em pacientes com doença renal crônica em hemodiálise. J Bras Nefrol. 2007; 29(4):222-9.

40. Castro M, Caiuby AVS, Draibe A, Canziani ME. Qualidade de vida de pacientes com insuficiência renal crônica em Hemodiálise avaliada através do instrumento genérico SF-36. Rev Assoc Med Bras. 2003;49(3):245-9.
Recebido: $14 / 12 / 2010$

Received: $12 / 14 / 2010$

Aprovado: $17 / 06 / 2011$

Approved: 06/17/2011 